\title{
Heart Failure With Preserved, Mid-Range, and Reduced Ejection Fraction
}

\author{
The Misleading Definition of the New Guidelines
}

\begin{abstract}
$T^{1}$ he European Society of Cardiology (ESC) recently published new guidelines for the diagnosis and treatment of acute and chronic heart failure (HF). ${ }^{1}$ The new nomenclature includes separating patients with HF into 3 distinct groups depending on the left ventricular ejection fraction (LVEF): preserved LVEF ( $\geq 50 \%$ ), mid-range LVEF (40-49\%), and reduced LVEF ( $\leq 40 \%)$. Although there have been several studies that argue for and against stratifying HF patients by LVEF, the latest guidelines continue to focus mainly on the LVEF as the central determinant of prognosis in HF. However, further characterization of HF phenotype using etiology, comorbidities, and nonresponse to therapy among the 3 proposed groups are not incorporated into the definition. It is important to identify pathophysiological mechanisms and specific etiologies that underlie the clinical status, beyond the simplistic definition of preserved, mid-range, and reduced LVEF. Moreover, the term "preserved" could be misleading and confusing: quite comforting and mistakenly reassuring. As observed in the literature and in clinical practice, patients with preserved LVEF may have worse prognosis in terms of rehospitalization and mortality. Furthermore, it is necessary to highlight that the determination of LVEF from 2D echocardiographic images with Simpson's biplane technique is relatively unreliable, with intra and interobserver variability of up to $13 \%$ and $15 \%$, respectively, because of foreshortened views and geometric assumptions. ${ }^{2}$ Moreover, LVEF calculation is sensitive to changes in hemodynamic loading conditions. This is what occurs in patients with mitral regurgitation who have preserved LVEF despite severe ventricular dysfunction., ${ }^{3,4}$ The consequence of such variability in measurement and sensitivity to loading conditions may lead to a significant overlap among the 3 proposed categories that are separated by only a few percentage points. Moreover, calculating LVEF is considered a simple method to estimate ventricular function, but in fact it may be too simplistic. In the management of HF patients, it is more important to focus on ventricular function estimated by chamber volumes and pressures, as well as by Doppler flows and tissue Doppler imaging (TDI). In fact, in our opinion, the key means by which to determine the prognosis of HF patients involves establishing the presence or absence of ventricular dysfunction, that could be (1) systolic, ie, with increased ventricular volumes; (2) diastolic, ie, with abnormalities in transmitral and pulmonary veins flows, in TDI mitral annular velocities, and in left atrium volume; or (3) systo-diastolic, ie, including features of both systolic and diastolic dysfunction. The presence of systolic, diastolic, or systo-diastolic ventricular dysfunction determines low cardiac output, which is the crucial pathophysiological element of HF.

The following example, which is not rare in clinical practice, demonstrates how important it is to define ventricular function more completely rather than to focus on LVEF alone. Consider a patient who presents with worsening dyspnea, but who also suffers from hypertensive heart disease with LV hypertrophy and high filling pressures, but with preserved LVEF, atrial fibrillation with poor rate control, renal impairment due to or enhanced by low cardiac output, and pulmonary infections due to preexisting chronic obstructive pulmonary disease. Such patients are routinely observed in the real world and tend to have a poor prognosis in terms of rehospitalization and mortality despite a preserved LVEF. ${ }^{5,6}$ In fact, patients with preserved or mid-range LVEF may present with a low cardiac output due to several mechanisms, such as modifications in loading conditions and the presence of arrhythmias like atrial fibrillation. Therefore, it is important to decipher pathophysiological mechanisms that underlie the functional status, beyond the simplistic definition of preserved, mid-range, and reduced LVEF. In the management of patients with HF, we believe that the assessment of ventricular function, which depends on chamber volumes and pressures, as well as on Doppler flows and TDI images, could be more accurate and useful than the mere identification of LVEF that is suggested by the latest ESC guidelines. Therefore, instead of utilizing the new ESC classification of preserved, mid-range, and reduced LVEF to stratify HF patients, as mentioned earlier, we propose an alternate classification scheme that takes into consideration LV dysfunction beyond LVEF, identifying 3 distinct categories: (1) systolic, (2) diastolic, and (3) systo-diastolic LV dysfunction.
\end{abstract}

Disclosure: The authors declare no conflict of interest.

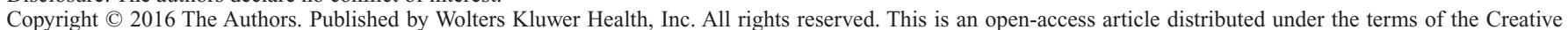
Commons Attribution-Non Commercial-No Derivatives License 4.0 (CCBY-NC-ND), where it is permissible to download and share the work provided it is properly cited. The work cannot be changed in any way or used commercially without permission from the journal. 
In our opinion, the current ESC guidelines for diagnosing and treating HF according to LVEF are misleading and confusing. We hope our more comprehensive assessment of ventricular function would be found more accurate and useful.

\section{REFERENCES}

1. Ponikowski P, Voors AA, Anker SD, et al. 2016 ESC Guidelines for the diagnosis and treatment of acute and chronic heart failure. Eur Heart J. 2016;18:891-975.

2. Wood PW, Choy JB, Nanda NC, et al. Left ventricular ejection fraction and volumes: it depends on the imaging method. Echocardiography 2014;31:87-100.

3. Gaasch WH, Meyer TE. Left ventricular response to mitral regurgitation: implications for management. Circulation 2008;118:2298-2303.

4. Berko B, Gaasch WH, Tanigawa N, et al. Disparity between ejection and end-systolic indexes of left ventricular contractility in mitral regurgitation. Circulation 1987;75:1310-1319.
5. Owan TE, Hodge DO, Herges RM, et al. Trends in prevalence and out come of heart failure with preserved ejection fraction. $N$ Engl J Med. 2006;355:251-259.

6. Meta-analysis Global Group in Chronic Heart Failure (MAGGIC). The survival of patients with heart failure with preserved or reduced left ventricular ejection fraction: an individual patient data meta-analysis. Eur Heart J. 2012;33:1750-1757.

Francesco Fedele, MD

Massimo Mancone, MD, PhD

Francesco Adamo, MD

Paolo Severino, MD

Department of Cardiovascular, Respiratory, Nephrology, Anesthesiology and Geriatric Sciences Sapienza University of Rome Rome, Italy 